Supporting Information For:

\title{
Documentation of an Imperative to Improve Methods for Predicting Membrane Protein Stability
}

Brett M. Kroncke ${ }^{\dagger}$, Amanda M. Duran ${ }^{\ddagger}$, Jeffrey L. Mendenhall ${ }^{\ddagger}$, Jens Meiler ${ }^{\star}$, Jeffrey D. ${\text { Blume } \|^{*} \text {, Charles R. Sanders }}^{\dagger * *}$

$\dagger$ Department of Biochemistry, †Center for Structural Biology, ${ }^{\S}$ Departments of Chemistry, Pharmacology, and Bioinformatics, and "Department of Biostatistics, Vanderbilt University, Nashville, Tennessee 37240, United States.

\section{Contents:}

Figures S1-S17

Separate File: Table S1: Excel-readable file with all experimental and calculated $\Delta \Delta G$ values: Table S1_ddg.csv

\section{Legends for Supporting Figures}

Figure S1. Comparison of Concordance, Pearson, and Spearman correlation coefficients for Rosetta based prediction methods that include membrane-specific score terms, ddg_monomer high-resolution protocol using RosettaMembrane, and low-resolution RosettaMPddG. As expected, this resulted in very similar correlations for RosettaMembrane (CC: 0.103, PC: 0.307, SC: 0.342 ) and Rosetta-High (CC: 0.11, PC: 0.28, SC: 0.37) as well as for RosettaMPddG (CC: 0.00, PC: 0.19, SC: 0.23) and Rosetta-Low (CC: 0.01, PC: 0.18, SC: 0.32).

Figure S2. Comparison of Concordance, Pearson, and Spearman correlation coefficients for $\beta$ barrel proteins.

Figure S3. Comparison of Concordance, Pearson, and Spearman correlation coefficients for $\alpha$ helical proteins.

Figure S4. Comparison of Concordance, Pearson, and Spearman correlation coefficients of point mutations that involve a proline or glycine.

Figure S5. Comparison of Concordance, Pearson, and Spearman correlation coefficients of point mutations that do not involve a proline or glycine.

Figure S6. Comparison of Concordance, Pearson, and Spearman correlation coefficients for residues in the aqueous phase.

Figure S7. Comparison of Concordance, Pearson, and Spearman correlation coefficients for residues at the interface between membrane and aqueous phases. 
Figure S8. Comparison of Concordance, Pearson, and Spearman correlation coefficients residues in the aliphatic phase of the membrane.

Figure S9. Comparison of Concordance, Pearson, and Spearman correlation coefficients for solvent exposed residues.

Figure S10. Comparison of Concordance, Pearson, and Spearman correlation coefficients for buried residues.

Figure S11. Comparison of Concordance, Pearson, and Spearman correlation coefficients for the bacterial proton pump, bacteriorhodopsin

Figure S12. Comparison of Concordance, Pearson, and Spearman correlation coefficients for glycophorin A

Figure S13. Comparison of Concordance, Pearson, and Spearman correlation coefficients for the E. coli rhomboid protease, GlpG

Figure S14. Comparison of Concordance, Pearson, and Spearman correlation coefficients for the disulfide formation protein B, DsbB

Figure S15. Comparison of Concordance, Pearson, and Spearman correlation coefficients for the outer membrane phospholipase A1, OmpLA

Figure S16. Comparison of Concordance, Pearson, and Spearman correlation coefficients for the outer membrane protein A, OmpA

Figure S17. Comparison of Concordance, Pearson, and Spearman correlation coefficients for the lipid A palmitoyltransferase, PagP 
Figure S1.

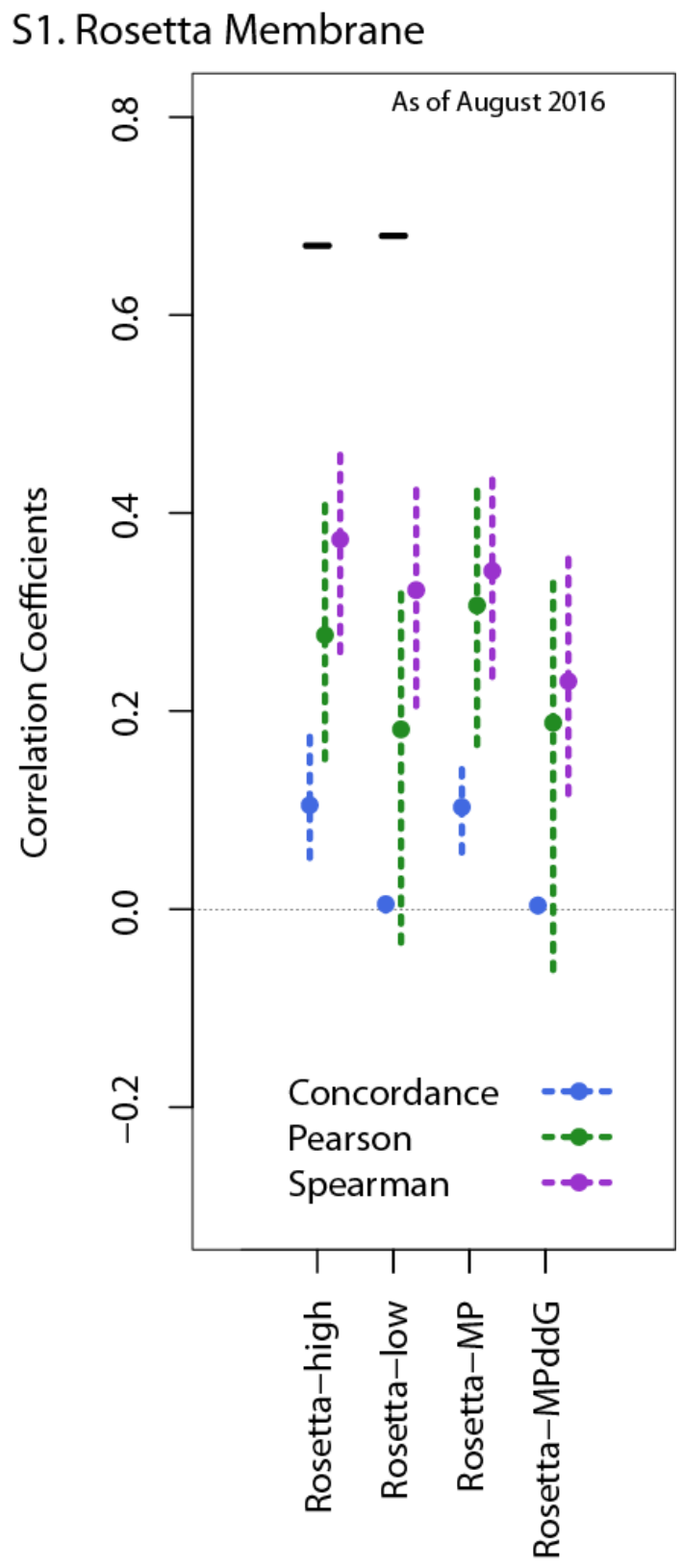


Figure S2.

\section{S2. Beta Barrel Proteins}

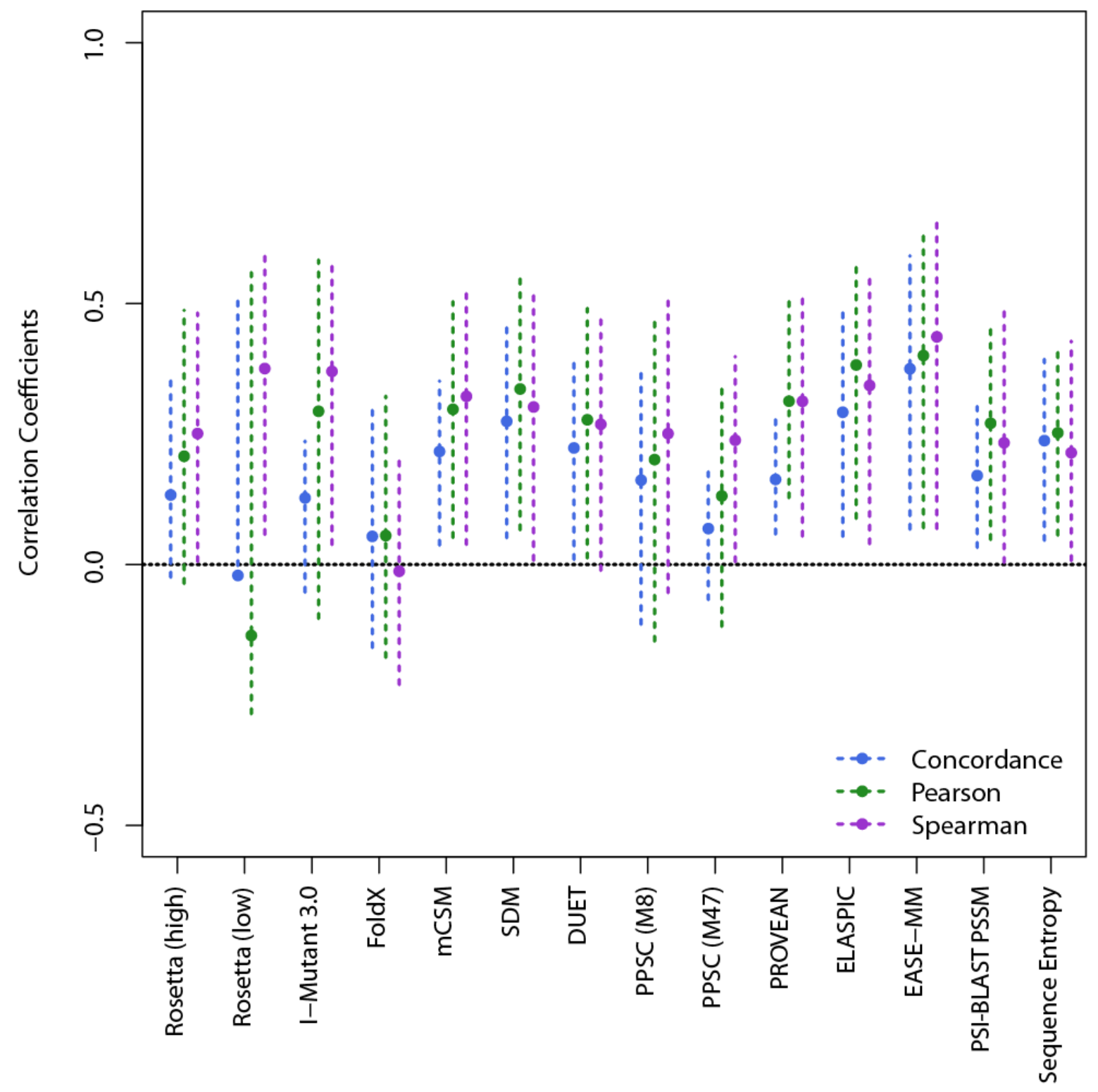


Figure S3.

\section{S3. Alpha Helical Proteins}

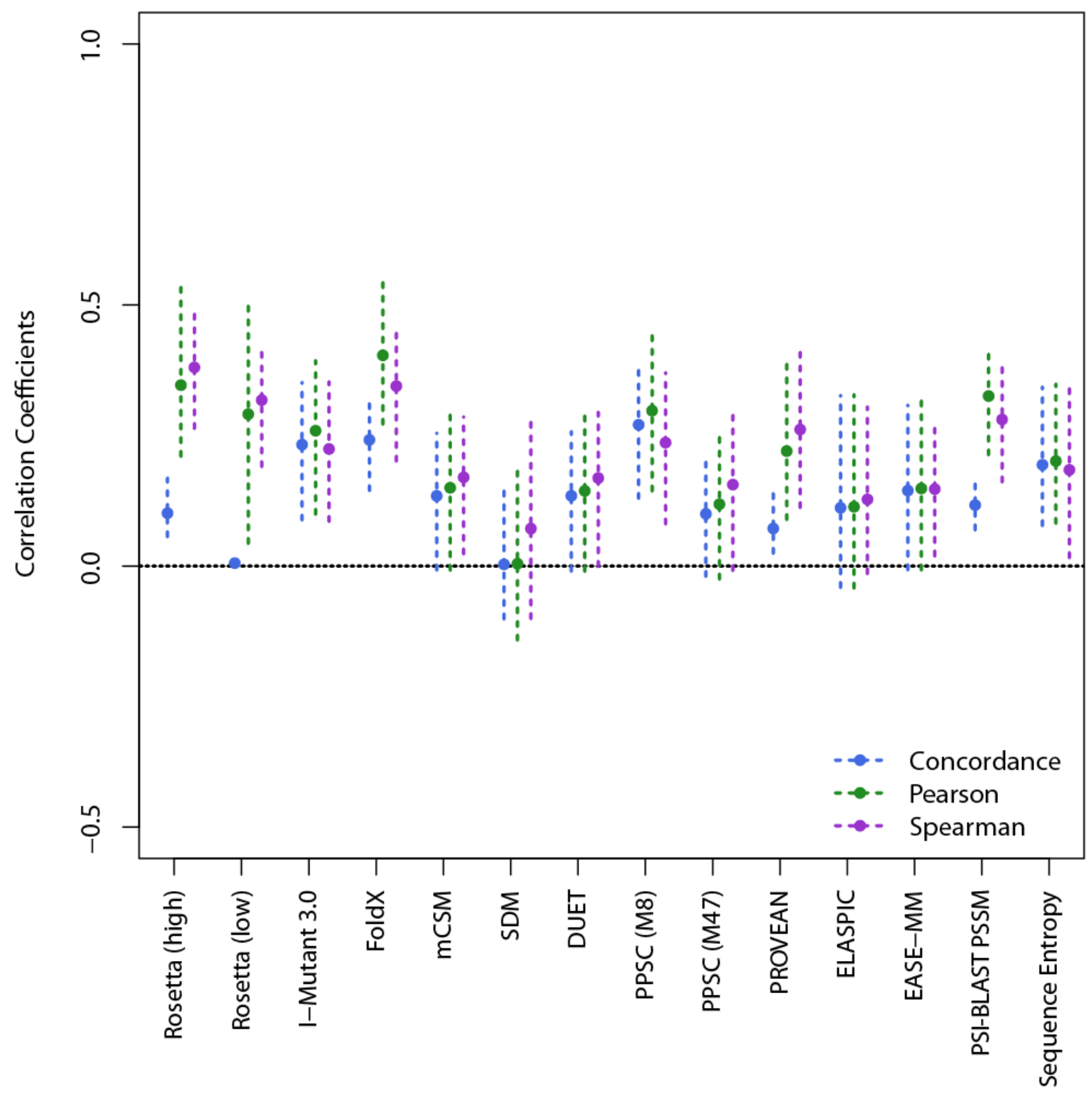


Figure S4.

S4. Residues involving a Glycine or Proline

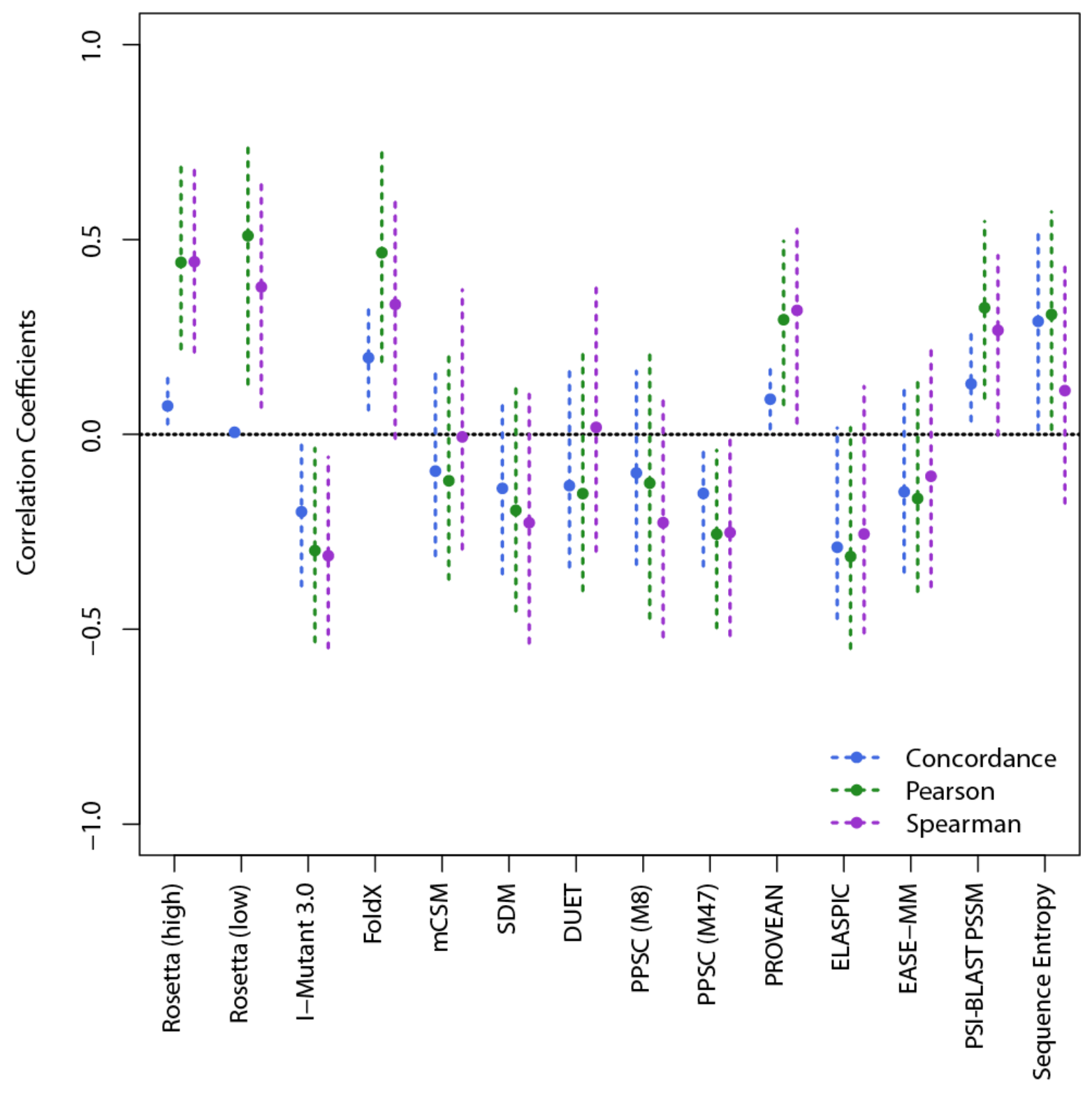


Figure S5.

S5. Residues not involving a Glycine or Proline

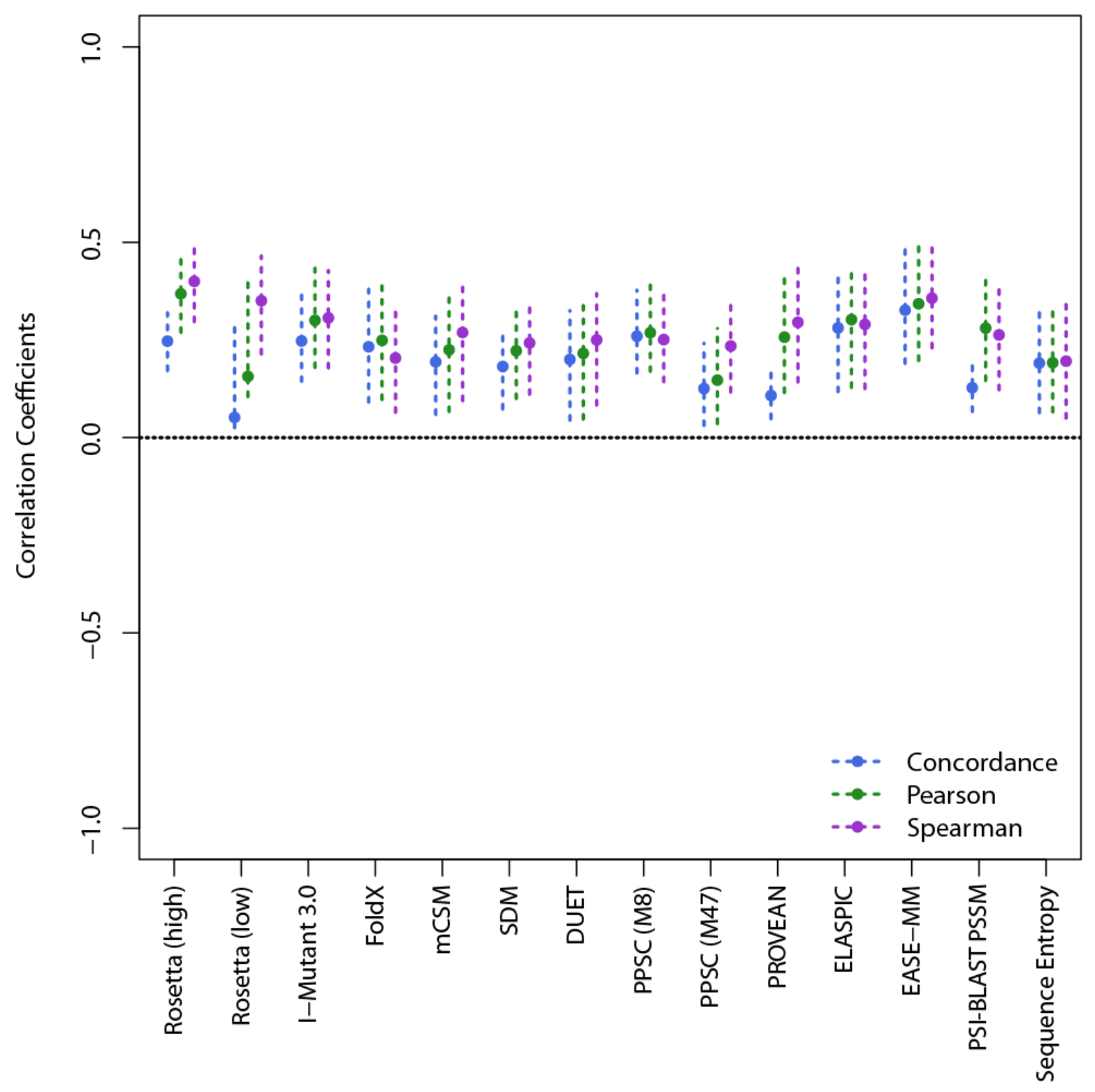


Figure S6.

S6. Residues in the Aqueous Phase

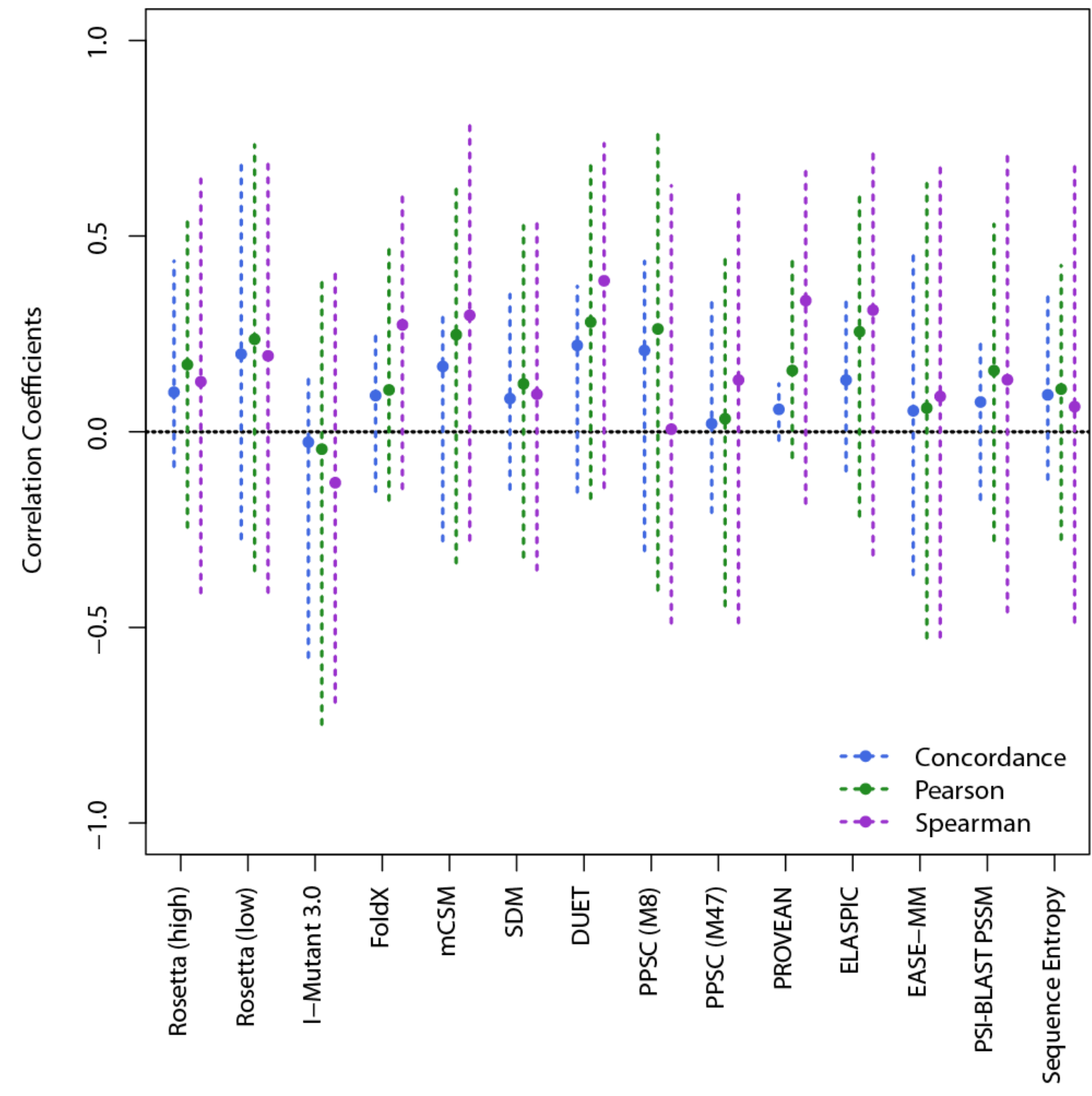


Figure S7.

S7. Residues at the Interface between Aqueous and Aliphatic Phases

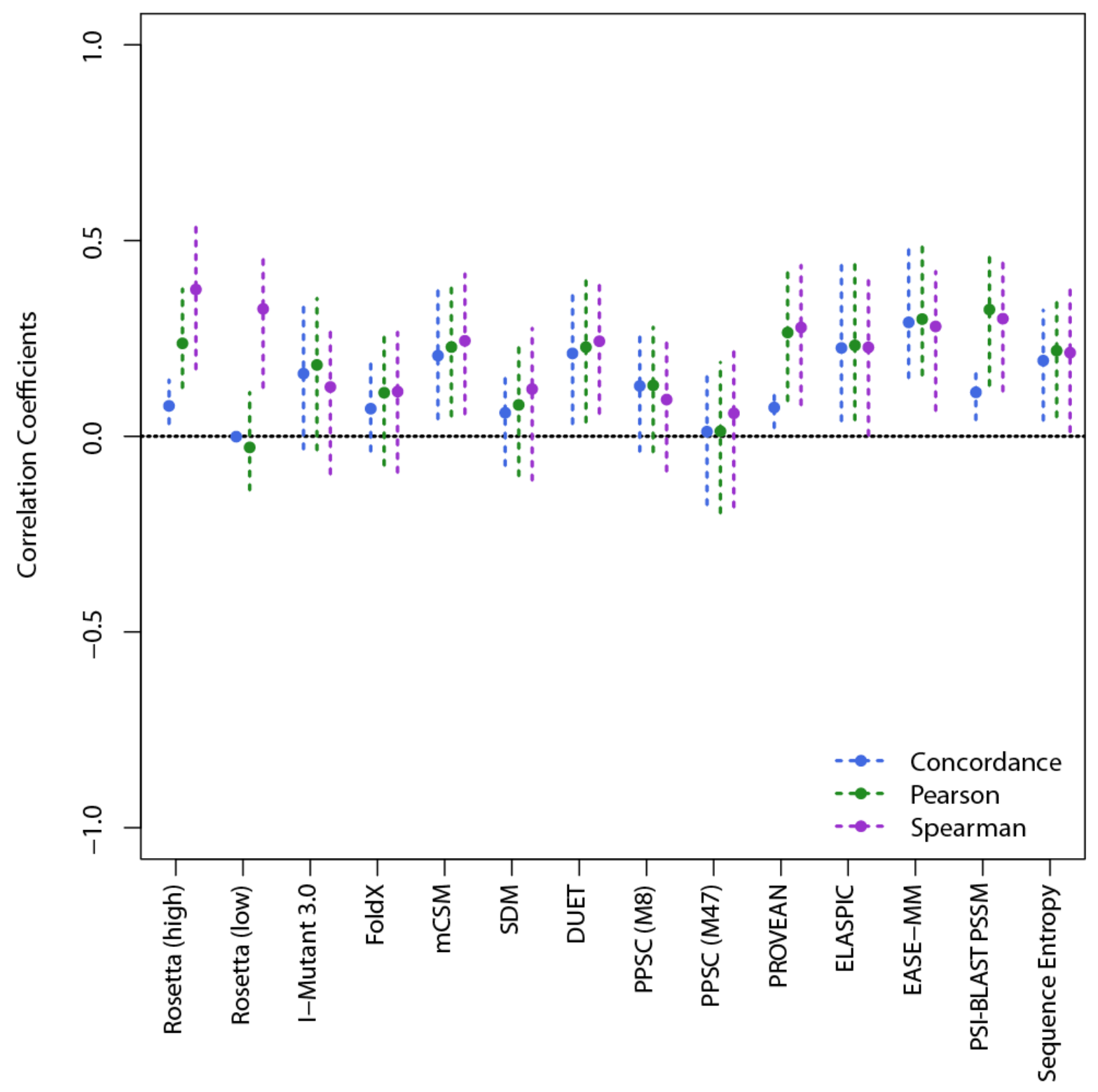


Figure S8.

S8. Residues in the Aliphatic Phase

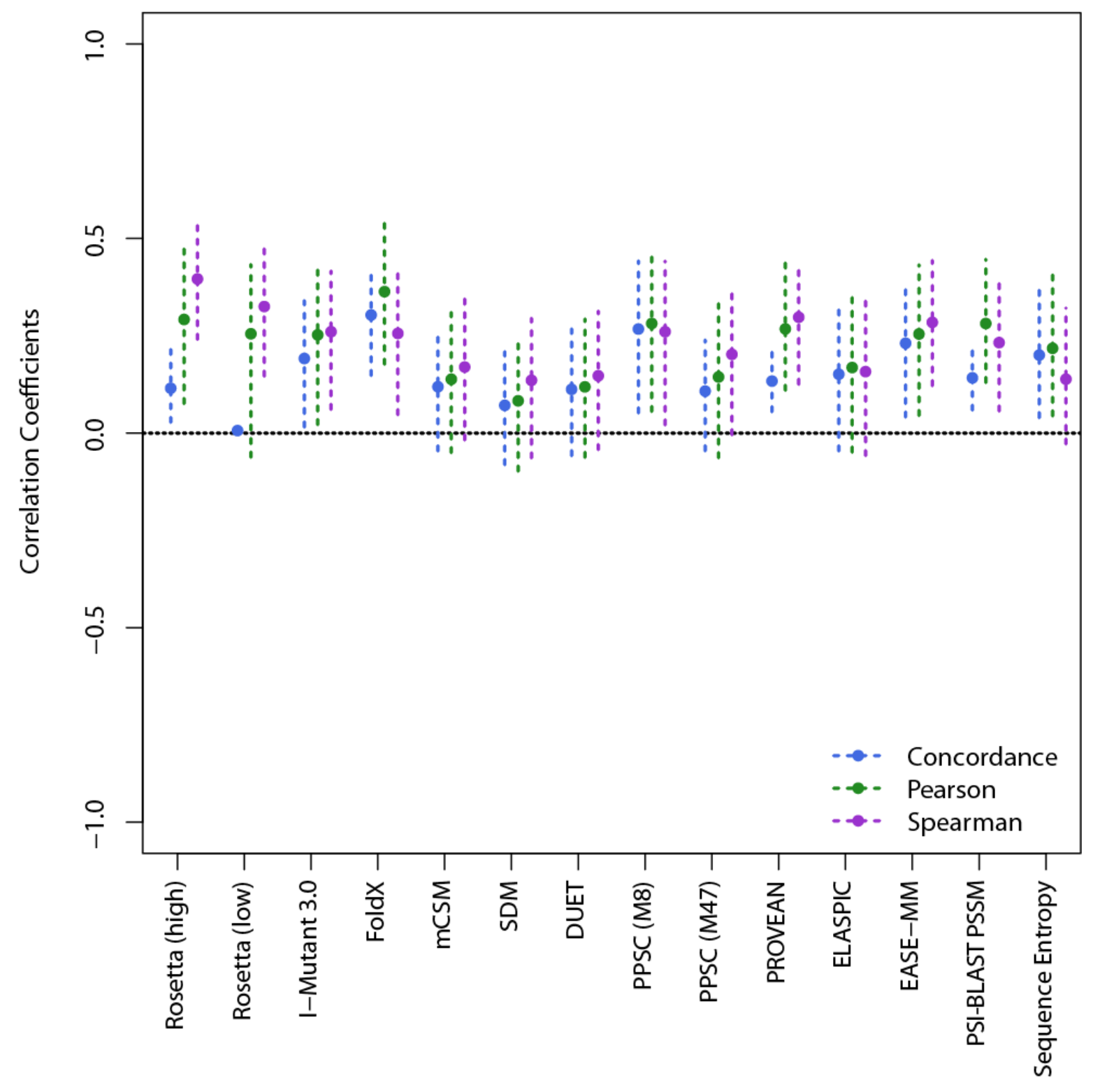


Figure S9.

S9. Residues Exposed to Solvent

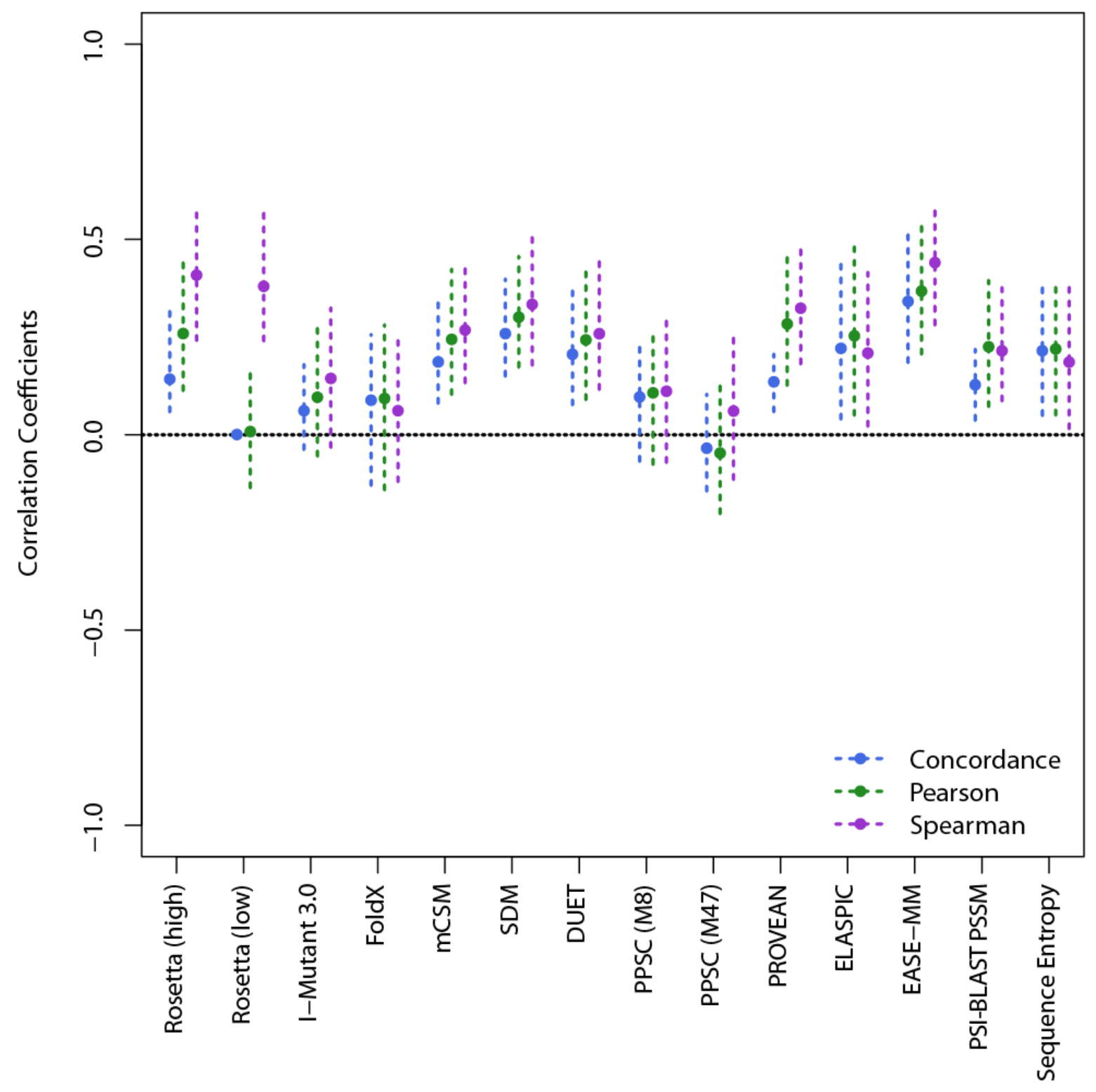


Figure S10.

S10. Residues Burried within the Protein

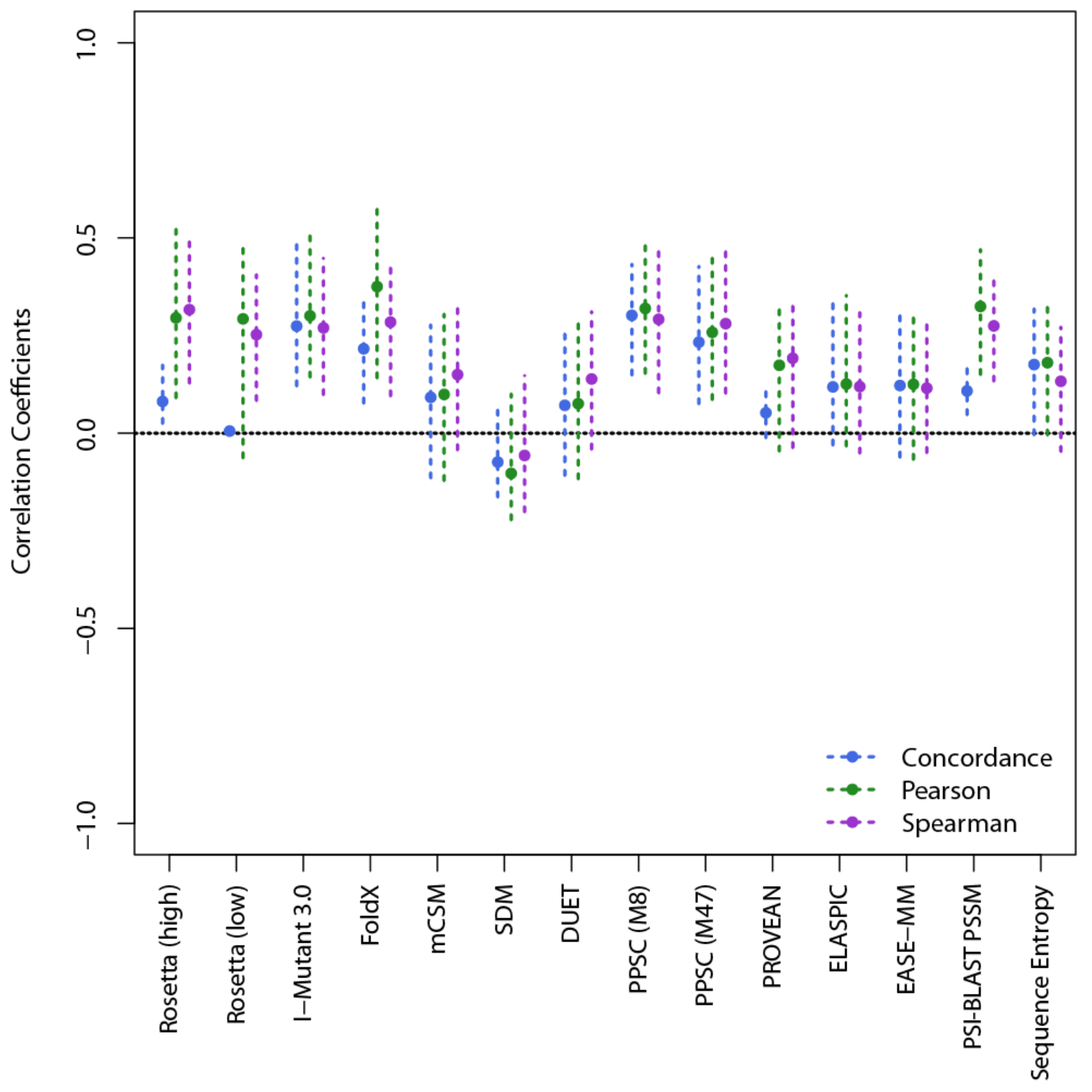


Figure S11.

S11. Residues in the Bacterial Proton Pump, Bacteriorhodopsin

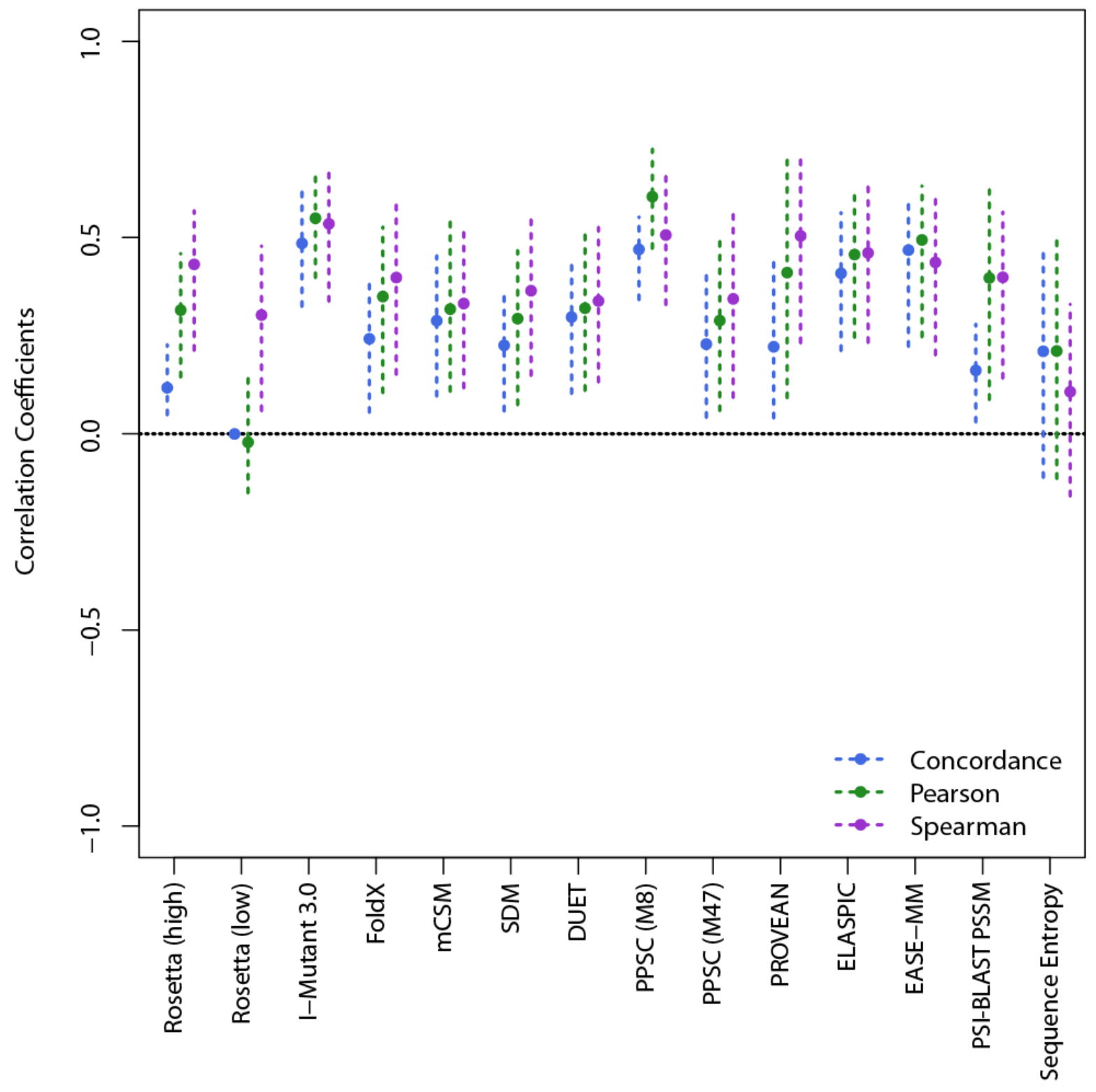


Figure S12.

S12. Residues in Glycophorin A

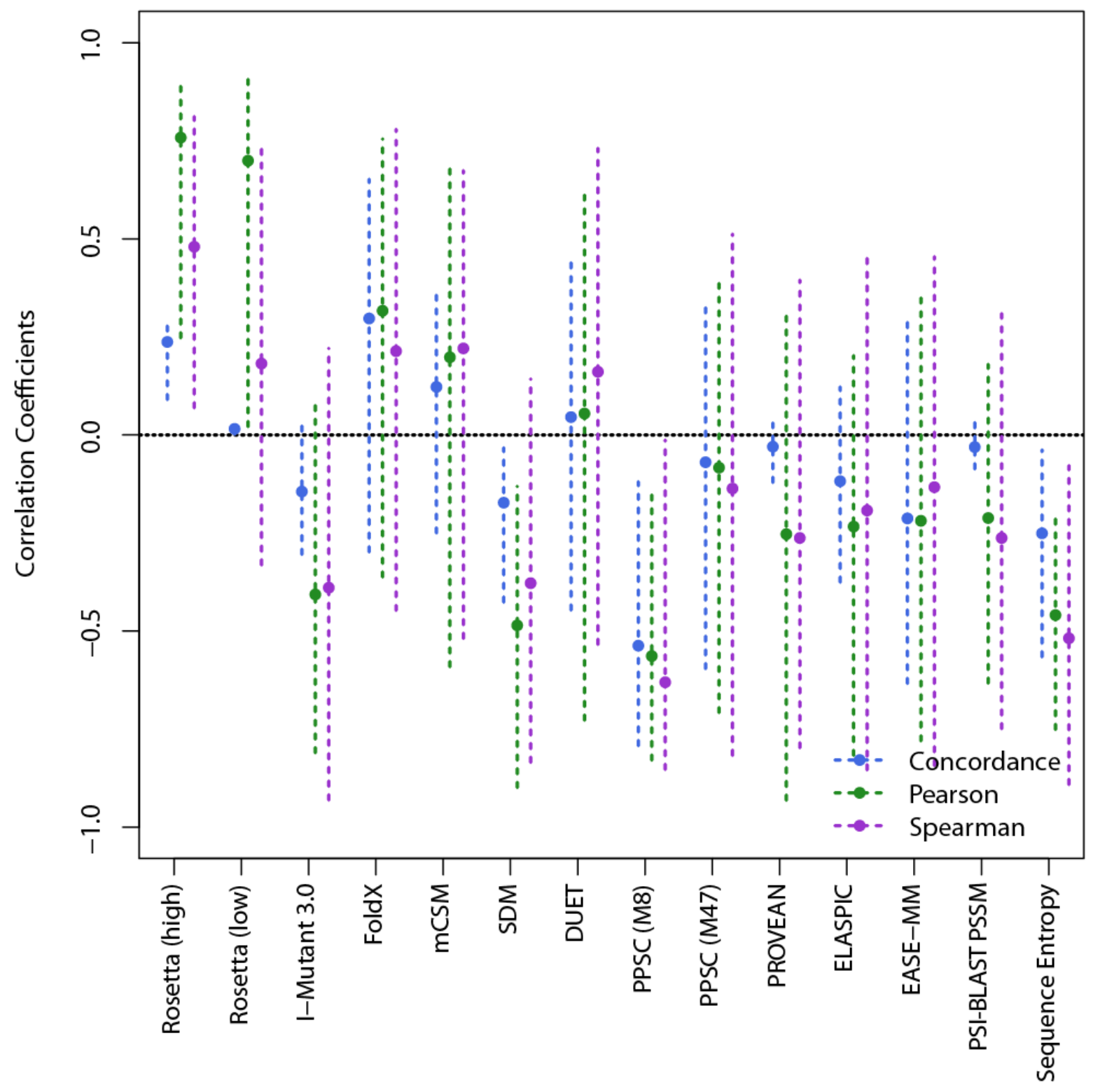


Figure S13.

S13. Residues in the the E. coli Rhomboid Protease, GlpG

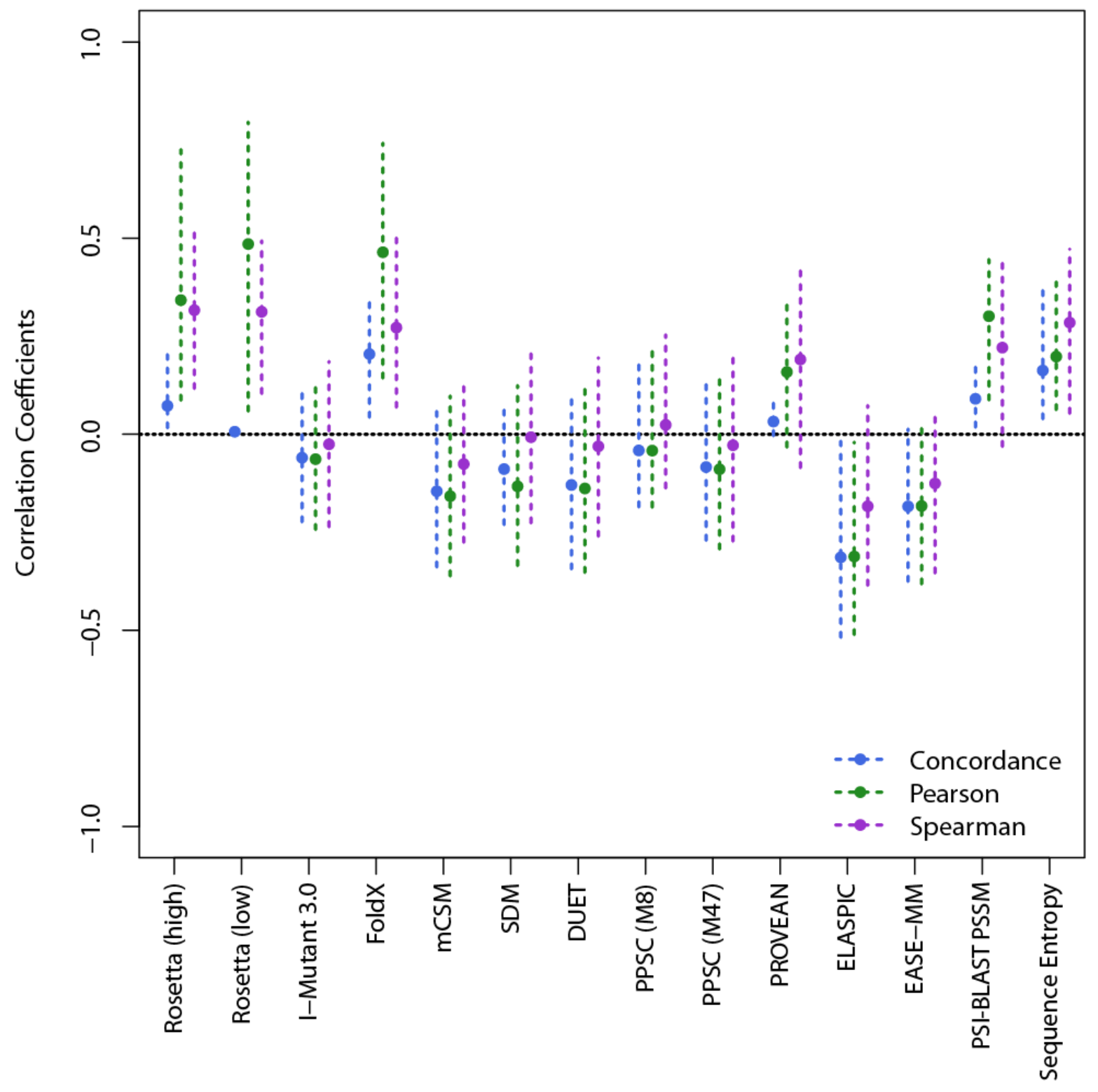


Figure S14.

S14. Residues in the the Disulfide Formation Protein B, DsbB

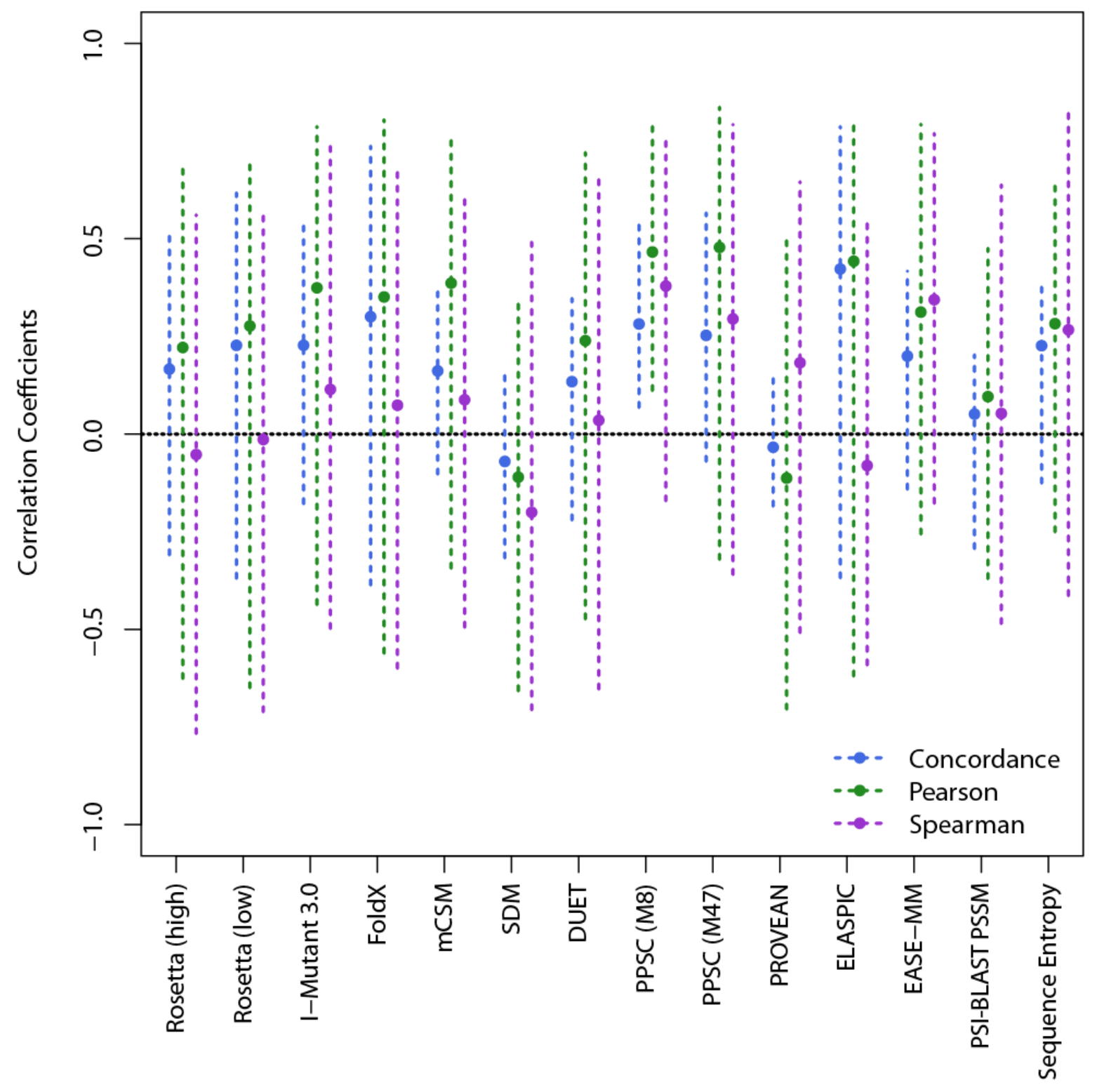


Figure S15.

S15. Residues in the the the Outer Membrane Phospholipase A1, OmpLA

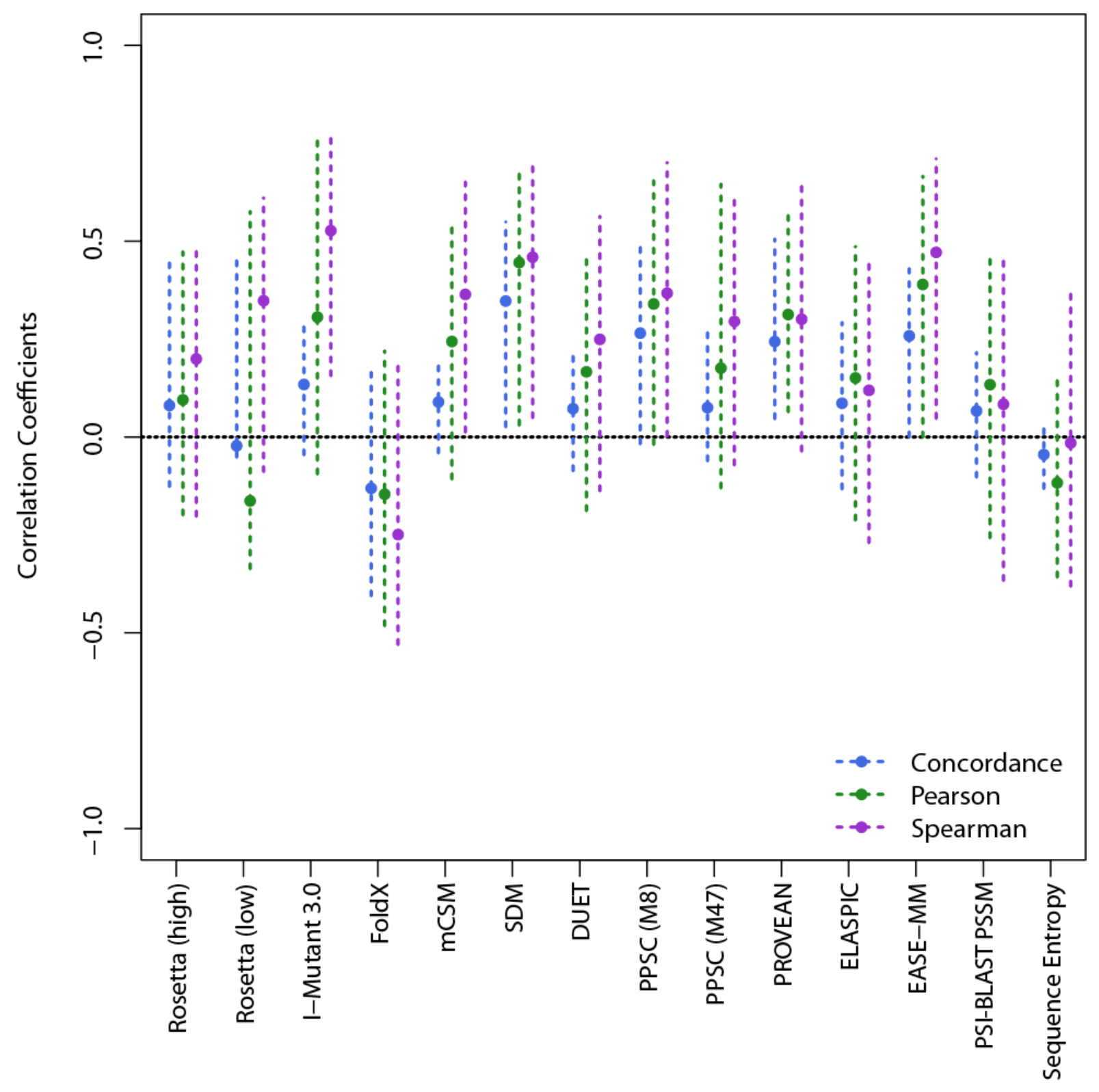


Figure S16.

S16. Residues in the the the the Outer Membrane Protein A, OmpA

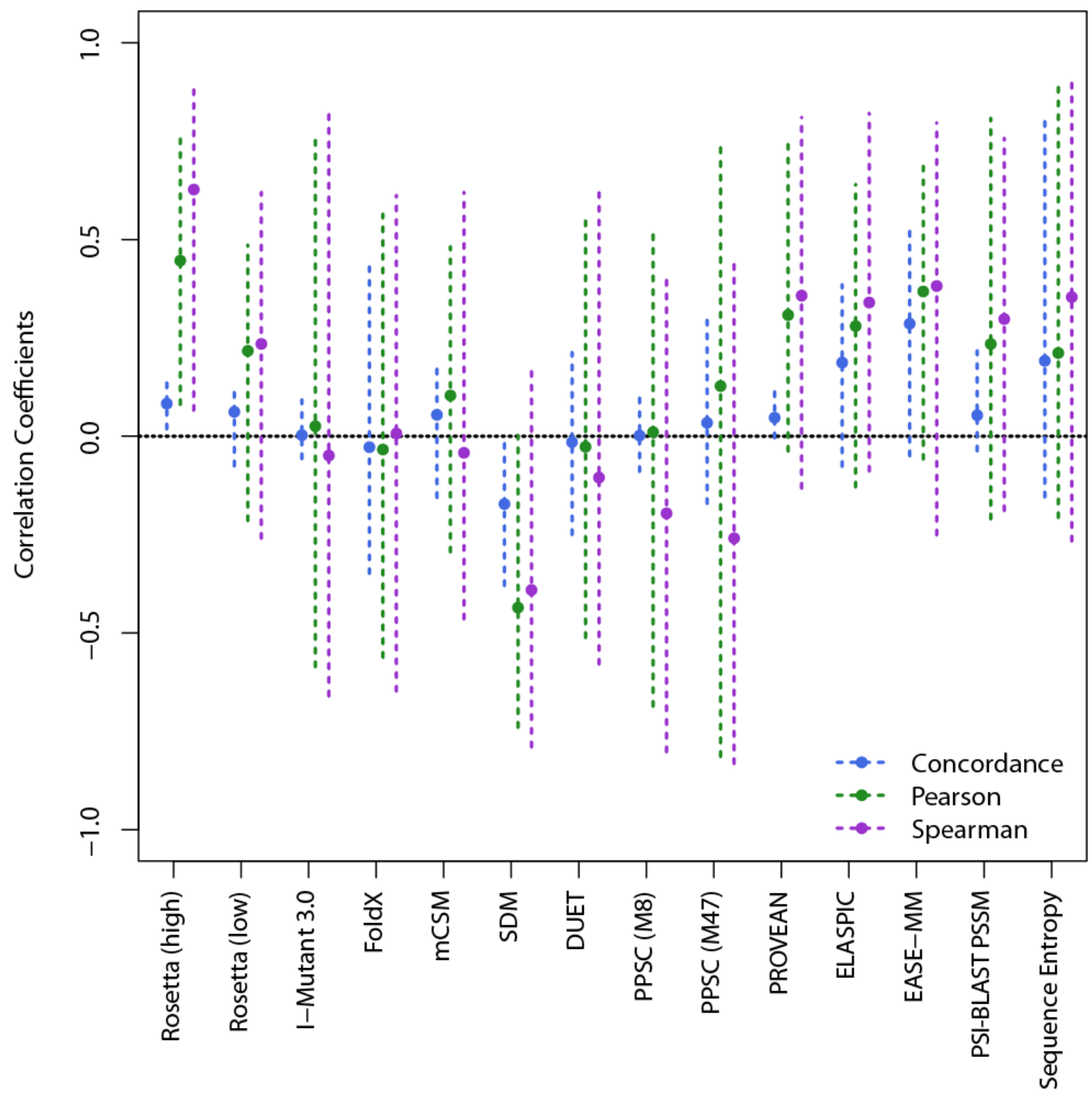


Figure S17.

S17. Residues in the the the the Lipid A Palmitoyltransferase, PagP

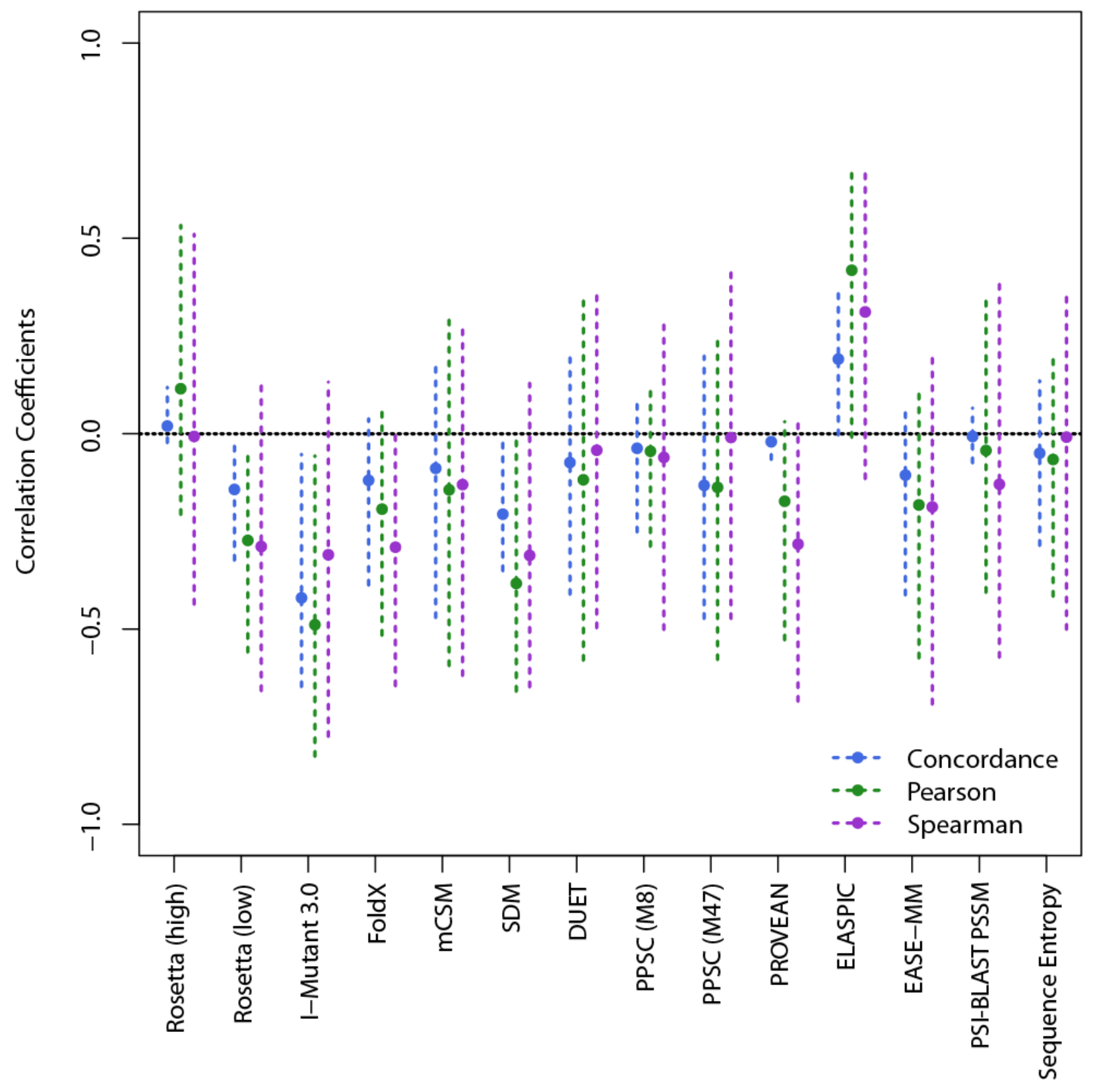

